\title{
Bacteria Pattern Spontaneously on Periodic Nanostructure Arrays
}

\section{Citation}

Hochbaum, Allon I., and Joanna Aizenberg. 2010. Bacteria Pattern Spontaneously on Periodic Nanostructure Arrays. Nano Lett. 10, no. 9: 3717-3721. doi:10.1021/nl102290k.

\section{Published Version}

doi:10.1021/nl102290k

\section{Permanent link}

http://nrs.harvard.edu/urn-3:HUL.InstRepos:27417439

\section{Terms of Use}

This article was downloaded from Harvard University's DASH repository, and is made available under the terms and conditions applicable to Open Access Policy Articles, as set forth at http:// nrs.harvard.edu/urn-3:HUL.InstRepos:dash.current.terms-of-use\#OAP

\section{Share Your Story}

The Harvard community has made this article openly available.

Please share how this access benefits you. Submit a story.

Accessibility 


\title{
Bacteria pattern spontaneously on periodic nanostructure
}

\section{arrays}

\author{
AUTHOR NAMES Allon I. Hochbaum, Joanna Aizenberg* \\ AUTHOR ADDRESS The School of Engineering and Applied Sciences, Harvard University \\ The Department of Chemistry and Chemical Biology, Harvard University
}

AUTHOR EMAIL ADDRESS jaiz@seas.harvard.edu

RECEIVED DATE (to be automatically inserted after your manuscript is accepted if required according to the journal that you are submitting your paper to)

\begin{abstract}
Surface-associated bacteria typically form self-organizing communities called biofilms. Spatial segregation is important for various bacterial processes associated with cellular and community development. Here, we demonstrate bacterial ordering and oriented attachment on the single-cell level induced by nanometer-scale periodic surface features. These surfaces cause spontaneous and distinct patterning phases, depending on their periodicity, which is observed for several strains, both gram positive and negative. This patterning is a general phenomenon that can control natural biofilm organization.
\end{abstract}

KEYWORDS nanobiointerface, bacteria, biofilm, self-assembly, nanoposts.

In their natural state, bacteria form biofilms that are closely associated with surfaces, usually at the solid-liquid or liquid-air interface. These communities are composed of many cells embedded within a 
polymeric organic matrix. The formation of these composite structures is of concern to industry because biofilms can grow thick enough to obstruct pipelines in a variety of environments such as oil refineries, paper mills, and heat exchangers. In medical settings, biofilms cause extensive damage by triggering the human immune response, releasing harmful endotoxins and exotoxins, and clogging indwelling catheters. Hospital-acquired, or nosocomial, infections affect roughly $10 \%$ of patients in the United States - about 2 million annually, causing nearly 100,000 deaths ${ }^{1}$. Specifically, Pseudomonas aeruginosa is a human opportunistic pathogen and one of the most common nosocomial infections in the lining of catheters and the lungs of cystic fibrosis patients ${ }^{2,3}$. These infections are difficult to treat because the biofilm protects its constituent cells from antibiotic attack. Developing biomedical materials that are resistant to biofilm formation would significantly reduce the rate of nosocomial infections and the costs associated with treating them. Many negative effects of bacterial colonization stem from the formation of biofilms as protective structures and the associated cooperative behavior of bacterial cells mediated by quorum sensing and other virulence factors. As such, a comprehensive understanding of the interactions of bacterial cells at interfaces may lead to more effective treatments or surfaces that resist biofilm growth.

Persistently bacteria-resistant materials are difficult to achieve by surface chemistry alone. Even if bacteria are unable to attach directly to a substrate, non-specific adsorption of proteins or secreted surfactants to the surface eventually masks the underlying chemical functionality ${ }^{4-6}$. On the other hand, the effects of topographical features on bacterial adhesion and subsequent biofilm formation are poorly understood. Surface structures may provide a more persistent form of interaction between bacteria and surfaces. Nature provides some clues to preventing microbial colonization of surfaces. For example, ship hulls constantly amass layers of algae and crustaceans, whereas materials with topographical features mimicking the skin of sharks, for instance, have exhibited a remarkable resistance to marine biofouling ${ }^{7}$.

Recent studies have demonstrated that the behavior of mammalian cells can be manipulated using 
only spatial and mechanical cues ${ }^{8-10}$. Various cellular processes, from apoptosis to proliferation and differentiation, are dependent on the spatial confinement of cells ${ }^{11}$, and even stem cell fate can be determined by the stiffness of the underlying growth substrate ${ }^{12}$. Bacteria also respond to mechanical cues from the environment. Indeed, surface attachment is an integral step in biofilm formation and precipitates chemical signaling pathways within and between bacterial cells ${ }^{13}$. Substrate stiffness, for example, has been suggested to affect the density of surface colonization ${ }^{14}$. The role that surface structures play in modifying bacterial attachment and subsequent behavior, however, is unclear ${ }^{15}$.

Biofilms contain a diversity of microbial phenotypes and form spatial patterns through cooperative organization at the macroscopic ${ }^{16}$ and microscopic level. The formation of biofilms at interfaces (liquidsolid, liquid-air) is directed by gradients of nutrients, oxygen, and signaling molecules. In response to surrounding environmental factors, biofilms develop anisotropically and differentiated phenotypes distinct from those of the planktonic state - segregate accordingly ${ }^{17-21}$. Topographical features can influence the arrangement and the resulting behavior of cells on surfaces and may affect biofilm development. Cellular interactions generally comprise diffusive compounds, such as quorum sensing molecules, from surrounding cells or environmental stresses, but can also include spatial signals. Some bacteria rely on physical interactions between neighboring cells for communication ${ }^{22}$, and several critical cellular processes, including division and external signal processing such as chemotaxis sensors, localize at the polar ends of the bacterium ${ }^{23}$. Therefore, disrupting the natural packing arrangement of cells within biofilms may influence some of the cooperative functions of these microbial populations. Spatial confinement has been used to modify the configuration of surface-associated cells to a limited extent $^{15,24,25}$, for example, and can alter the threshold for biochemical reactions ${ }^{26,27}$. Here we report on periodic arrays of high-aspect-ratio nanostructures, which direct the large-scale spontaneous patterning behavior of bacteria. The configuration of cells on these surfaces is sensitive to the spacing between neighboring features and the bacterial patterns register precisely with the symmetry of the underlying array. Moreover, the effect is general and is observed in biofilm mutants and both gram-positive and 
gram-negative species.

To study the effects of substrate topography on bacterial ordering and biofilm development, nanostructured substrates were fabricated with dimensions on the order of bacterial cells. Arrays of high aspect-ratio nanometer-scale polymer posts were generated using a fast replication molding technique described previously ${ }^{28}$. Using this method, many identical substrates with varying dimensional parameters, such as nanopost diameter, height, pitch, and array symmetry, were made to conduct systematic investigations of bacterial growth on structured surfaces. These substrates were sputtercoated with thin layers of PtPd or AuPd to reduce the autofluorescence background and to provide a compatible surface for thiol chemical functionalization. As a result, the surface chemistry, substrate stiffness, post dimensions and symmetry could be tuned to explore a wide range of interaction parameters.

Pseudomonas aeruginosa (strain PA14), a rod-like gram-negative bacterium, was grown on submerged polymer replicas with a gradient of post pitch, from 4 down to $0.9 \mu \mathrm{m}$. The posts had a diameter of about $300 \mathrm{~nm}$, were $2 \mu \mathrm{m}$ tall, and arranged in an array with square symmetry. As opposed to the random packing and three-dimensional growth of biofilms on flat substrates, bacteria grown on these post substrates spontaneously assemble into patterns dictated by the underlying array symmetry (Figure 1). The fluorescence image in Figure 1a shows the interface between a flat region (upper) and one of patterned posts (lower) on the same substrate, upon which PA14 was cultured for $22 \mathrm{hr}$. in a rocking LB culture (see supporting information). The dissimilar ordering at the microcolony stage of biofilm formation is apparent, and the abrupt change at the interface suggests a localized response to topographical features rather than an induced cooperative behavior. Through-focusing fluorescence microscopy of substrates showed that bacteria attached at the post tips and overgrew of the array at longer incubation times. The basal layer of cells, however, filled the array completely and always retained the packing configuration shown in Figure 1a. The SEM images (Figure 1b, c) show crosssectional views of the different bacterial conformations in a biofilm grown on a flat substrate (Figure 
1b) and the extreme ordering case where cells are oriented normal to the substrate (Figure 1c). As is evident from the micrographs, the bacteria exhibit a preference for adhering to the posts even when different conformations are possible. This behavior was observed on such post substrates irrespective of surface chemistry and with and without the sputtered metal coating.

The spontaneous patterning of bacteria within the post arrays is extremely sensitive to the spacing between adjacent posts (i.e. the pitch minus the post diameter). Fluorescence microscopy images (Figure 2a-c), and the corresponding fast Fourier transforms (FFTs, Figure 2d), show the range of ordering achieved within the arrays over large areas. The FFTs contain both peaks associated with positional ordering of the bacteria and shape variations of the diffuse central spot, indicative of orientational order. The bacterial assembly is more pronounced as the spacing of the posts approaches the characteristic size of the cell. On regions of the sample where the nearest neighbor post spacing is larger than the length of the cell, adhesion of bacteria to the substrate is random (Figure 2a). The FFT from these areas shows no orientational order, akin to growth on flat unstructured surfaces and only faint positional ordering peaks, indicative of the preference of the cells to adhere at points where the posts meet the substrate.

As the spacing between neighboring posts approaches the length of the rod-like $P$. aeruginosa (roughly 1.2-1.5 $\mu \mathrm{m}$ ), bacteria adhere to the substrate in registration with the post array. Cells bridging nearest neighbor post positions - attach parallel to the substrate and perpendicular to each other - are aligned with the [10] and [01] directions of the post lattice. The FFTs in Figure 2d show this transition as the post pitch decreases. The central spot of the FFTs extends towards the [10] and [01] ordering peaks, indicative of the preferential alignment of the cells on the substrate. As the post spacing decreases further across the substrate, to about $0.8 \mu \mathrm{m}$, the bacteria align themselves along the length of the posts, normal to the substrate. Since the cells are oriented along the imaging axis of the microscope, the bacteria appear as dots (Figure 2c) - as opposed to rods (Figure 2b) - arranged in a square array. The FFT marks this transition with the loss of orientational order in the central spot, since the cells are radially symmetric in this configuration in plan view. The positional ordering peaks are retained due to 
the persistent association of the cells with the posts. Throughout the transition from disordered to ordered adhesion, these positional ordering peaks move further from the center of the FFT, consistent with the decreasing lattice spacing of the posts. All the cells assembled within the arrays tend to pack with the same configuration at a given post spacing. In areas of the substrate between these regions, the bacteria pack in a mixture of the two flanking ordering phases. As discussed above, the basal layer of cells retained these different packing phases at longer incubation times or higher seeding densities that led to biofilm overgrowth of the post array. Experiments are ongoing to establish the effects of patterning on biofilm development and properties.

The distinct packing arrangements of bacteria on periodic nanostructures are a result of a preferential association between the bacterial cells and the substrate. Live imaging of bacteria grown on these nanopost arrays indicates that they move freely, not only in and out of the spaces between posts, but also between neighboring positions within the array (supplementary information). Along with the faint positional ordering peaks in the FFTs of widely spaced posts and SEM images of cells packing within the post arrays (supplemental information), these observations indicate that the bacteria tend to maximize their contact area with the surface, where the posts act as topographical extensions of the substrate. This simplistic model predicts the packing arrangements of bacteria observed by SEM and fluorescence microscopy (Figure 3a). The scheme also predicts the existence of an intermediate ordering phase, around $1.2 \mu \mathrm{m}$ spacing for $P$. aeruginosa (or rod-like cells with comparable dimensions), where the cells could optimize their surface contact by adopting a diagonal orientation along the [11] directions of the square array. Indeed, this state is observed, where the cells are still perpendicular to each other but diagonal within the post array, as seen in the FFT by the increase in intensity of the [11] positional ordering peaks and slight extension of the central spot towards these peaks (Figure $3 \mathrm{~b}, \mathrm{c}$ ).

Bacteria adhere to surfaces by specific mechanisms, which can vary between species ${ }^{13}$. P. aeruginosa uses cellular appendages such as pili and flagella to find, attach to, and move about on surfaces. To investigate the relevant mechanisms by which PA14 assembled within the post arrays, mutants lacking 
the necessary genes to synthesize either pili or flagella were grown on identical post substrates to the wild type strain. The assembly of these strains at different post pitches is shown in Figure $4 a$ and $b$, respectively. The overall assembly is unchanged and characteristically similar to the wild type assembly. The lack of effect of the appendage knockouts on the assembly phenomenon indicates that a different mechanism is involved in bacterial affinity for the surface than for normal biofilm development. In addition, the flagellum knockouts tended to adhere and proliferate at a slower rate than either the wild type or pili mutants, which is consistent with the known adsorption mechanism of $P$. aeruginosa $^{13}$. Control experiments on the same substrates also confirmed that the patterning is not an effect of shear flow or sedimentation. Identical spontaneous assembly was observed on rocking and static cultures, and on inverted submerged substrates with the same pitch gradient as those shown in the figures. These results suggest that a biological, rather than physical, mechanism is responsible for the patterning behavior of the bacteria.

Other bacterial strains also use different genetic pathways and cellular appendages to adhere to surfaces. Wild type strains of both Bacillus subtilis (strain 3610) and Escherichia coli (strain W3110) were grown on the substrates with a gradient of post pitches, and similar cellular patterning was observed (Figure $4 \mathrm{c}$ and d). These disparate species assemble into patterns dictated by the post array and exhibit the similar ordering phases to PA14. One significant difference between the assemblies of these species on the same post pitch gradient substrates is the inter-post spacing at which the patterns form. Specifically, the spacing between neighboring posts at which bacteria began to order on the substrate correlates to the size of the cells. $B$. subtilis and $E$. coli cells ordered in subsequently larger gaps than $P$. aeruginosa, suggesting that the assembly phenomenon is related to interactions with the cell surfaces or biofilm components closely associated with the cell wall. Moreover, the patterned assembly is a general phenomenon, occurring in examples of both gram-positive and gram-negative bacteria, even in the absence of pili or flagella.

In summary, we have shown that when the characteristic dimensions of confined spaces approach 
those of bacterial cells, their interactions with the surface changes significantly. These interactions are general and apply to a variety of bacterial species, and they may be extended to other microorganisms such as fungi and marine microbes. Tuning the periodicity of structures within the relevant cellular scale leads to distinctive differences in bacterial assembly. In this manner, we have demonstrated the ability to direct cell patterning over large areas on a microscopic level. Furthermore, various substrate parameters, such as mechanical stiffness, surface chemistry, and feature size and spacing can be tuned independently to systematically investigate different aspects of bacterium-surface interactions and reveal developmental pathways in bacterial community. In this way, these substrates could elucidate new targets for antibiotic action or provide a novel route to engineer ordered or disordered biofilm structures for a variety of applications ranging from microbial-resistant surfaces that interfere with biofilm development by disrupting the natural packing arrangement of cells within biofilms to those that promote specific biofilm functions such as in remediation or bioelectrical systems.

ACKNOWLEDGMENT We thank Dr. Win Chai, Dr. Moshe Shemesh, Dr. Hera Vlamakis, Prof. Roberto Kolter, and Prof. Richard Losick for use of their facilities, access to various bacterial strains, and helpful discussions. The authors also thank BASF for funding.

Supporting Information Available: bacteria growth protocols, SEM images of patterned bacteria, video of PA14 cultured on patterned substrates. This material is available free of charge via the internet at http://pubs.acs.org. 


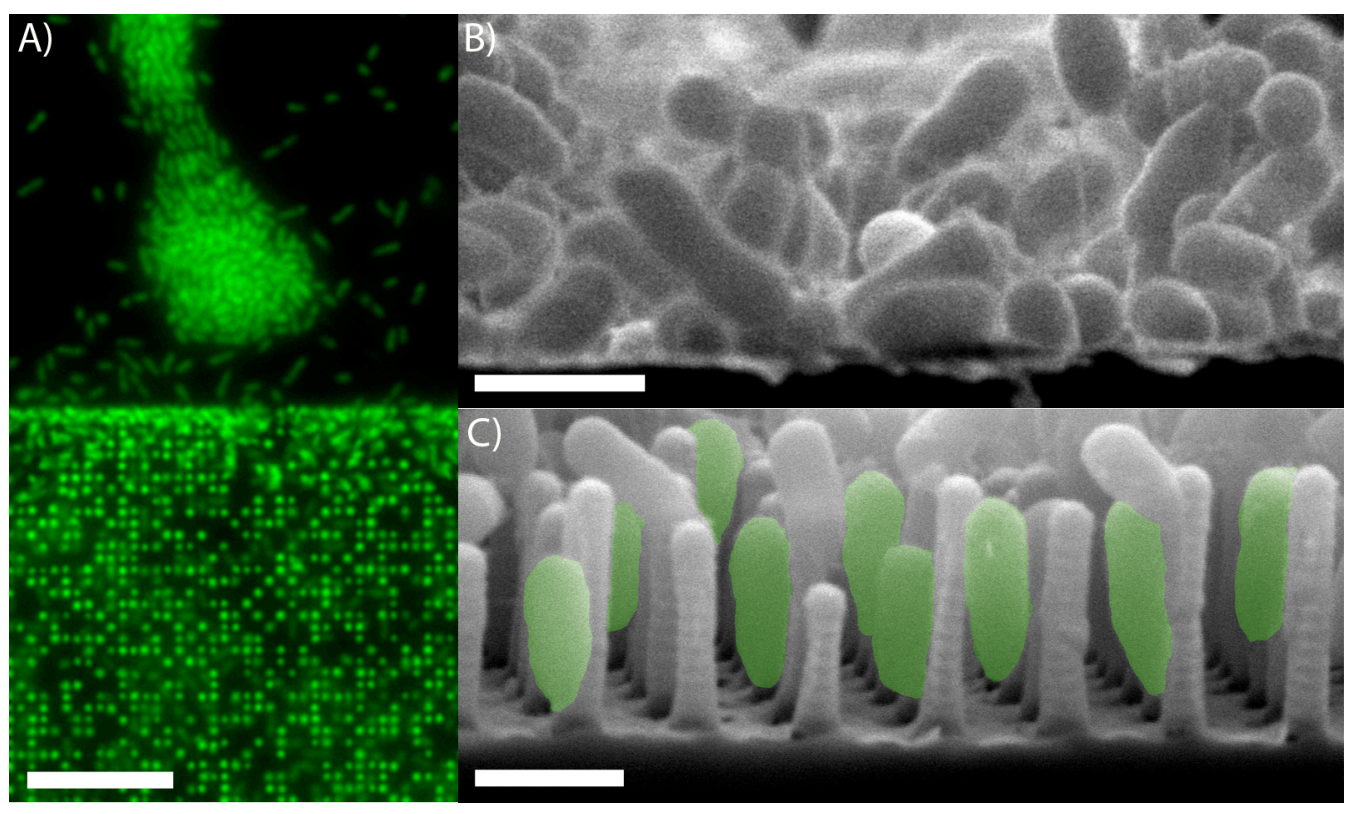

Figure 1. Comparison of $P$. aeruginosa adhesion on structured and unstructured regions of the growth substrates. A) Fluorescence microscopy shows the localized effect of substrate topography on bacterial adhesion as compared to flat surfaces. The image shown in $\mathbf{A}$ ) is of the interface between a structured and unstructured region on the same substrate. The interface between the flat (upper) and structured (lower) areas is abrupt, as is the transition from ordered packing to random microcolony aggregates, which lack long-range cell order. B) and C) are cross-sectional SEM images of PA14 cultured on flat and periodically structured epoxy surfaces, respectively, showing the stark difference in attachment morphology. The aligned cells in $\mathbf{C}$ ) are false-colored to highlight their orientation. Scale bars are 10 $\mu \mathrm{m}$ in $\mathbf{A}$ ) and $1 \mu \mathrm{m}$ in $\mathbf{B}$ ) and $\mathbf{C})$. 


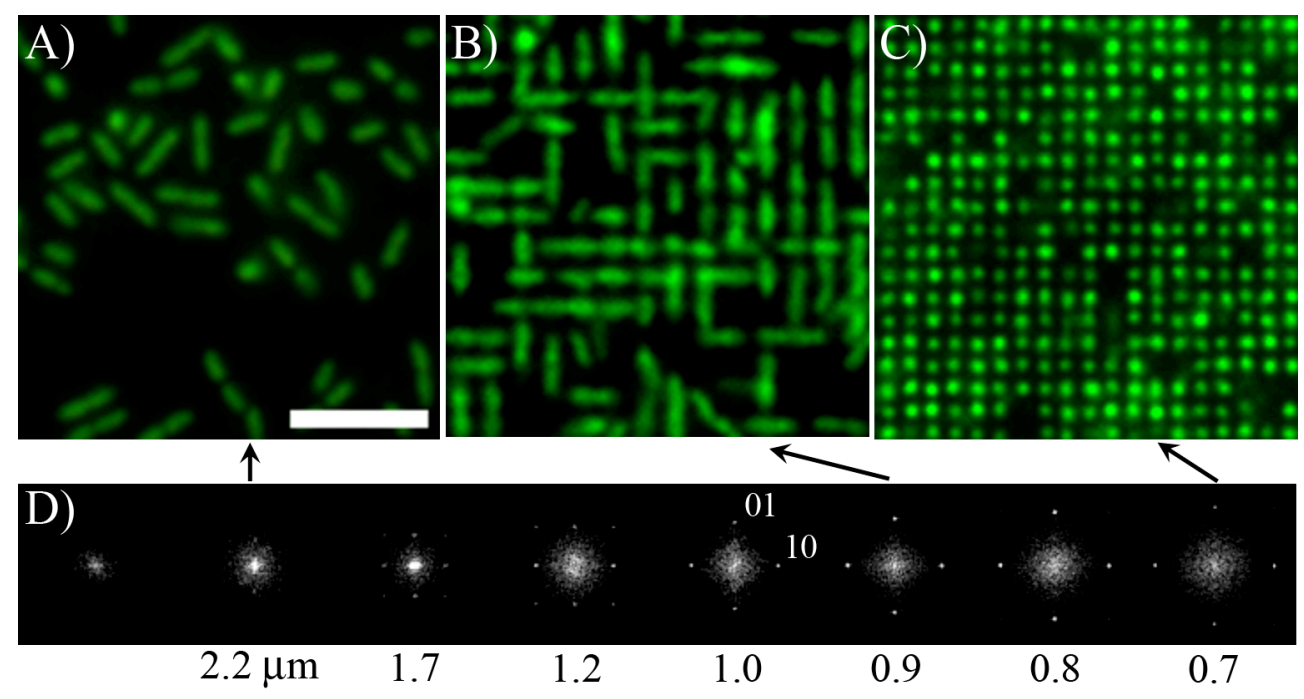

Figure 2. P. aeruginosa assembled on nanopost arrays. Fluorescence microscopy images of assembled bacteria on a post pitch gradient substrate at $2.2(\mathbf{A}), 0.9(\mathbf{B})$, and $0.7 \mu \mathrm{m}(\mathbf{C})$ spacing between posts show the different packing configurations of rod-like bacteria within the periodic arrays. D) FFTs of these and intermediate post spacing regions elucidate the ordering of cells on varying topographies. The FFT farthest to the left is from a flat substrate for comparison. The rest of the FFTs are from large area images of bacteria adhered to regions with decreasing post spacing (labeled under each FFT) from left to right. They all show positional ordering peaks corresponding to the [01] and [10] directions of the post array, indicating the preferential attachment and the subsequent registration of the bacterial layer with the posts. Scale bar in $\mathbf{A}$ ) is $5 \mu \mathrm{m}$ and applies to $\mathbf{B}$ ) and $\mathbf{C}$ ). 
A)
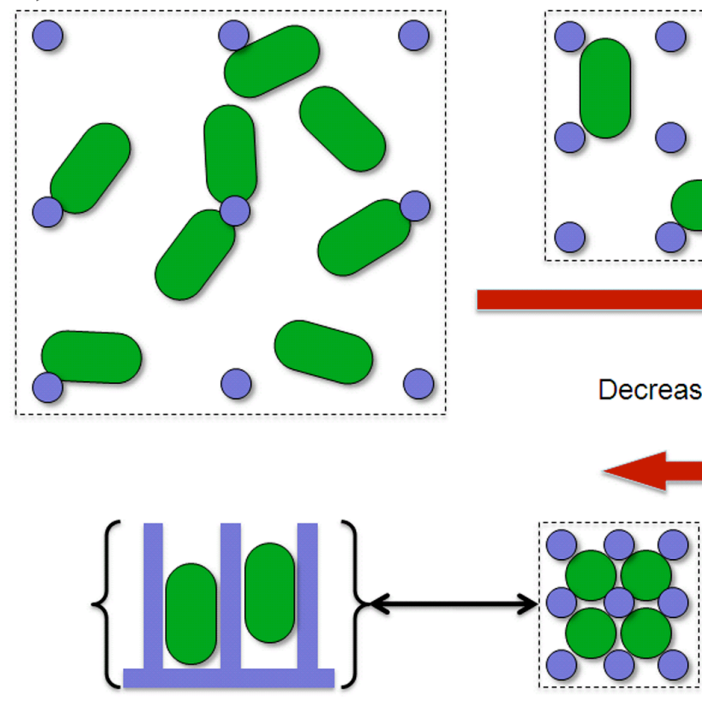

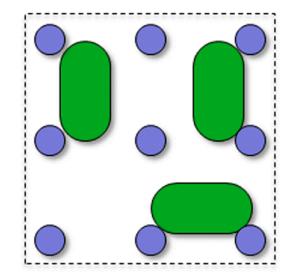

Decreasing post spacing

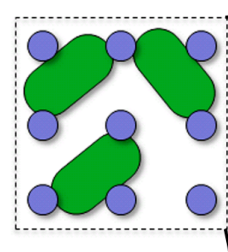

B)

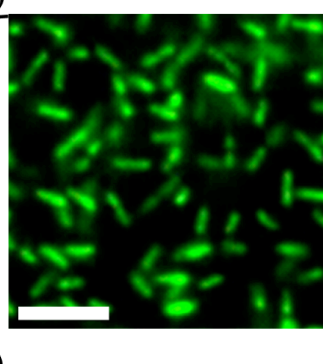

C)

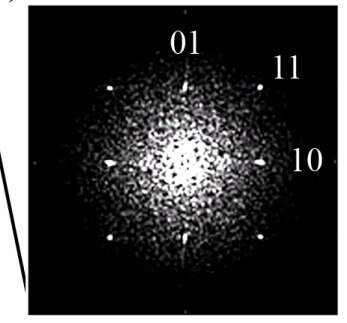

Figure 3. Maximization of surface contact area creates patterns in adhered bacteria. A) Plan view schematics of the adhesion patterns in rod-like bacteria on the nanopost arrays. The patterned assembly of these bacteria can be predicted by maximizing their surface contact area with the substrate. Clockwise from the top left, as the post pitch decreases, bacteria can increase their contact area by adhering to the increasing number of posts per area projecting normal to the substrate. In their most densely packed state, the cells orient normal to the substrate to maximize their contact with the posts (bottom left schematic is a cross-sectional view of the same conformation in the bottom middle). This model predicts an intermediate phase (top right) where cells can contact more posts by lying along the [11] directions of the square array or posts. This phase has been observed with fluorescence microscopy (B) at about $1.2 \mu \mathrm{m}$ post spacing for P. aeruginosa and the FFT of a large area image (C) confirms this orientation as evidenced by the [11] peaks and diagonal smearing of the center spot (note that neither the (11) peaks nor the diagonal smearing is observed in other packing phases on the substrate, see Figure 2). The scale bar in $\mathbf{B}$ ) is $10 \mu \mathrm{m}$. 

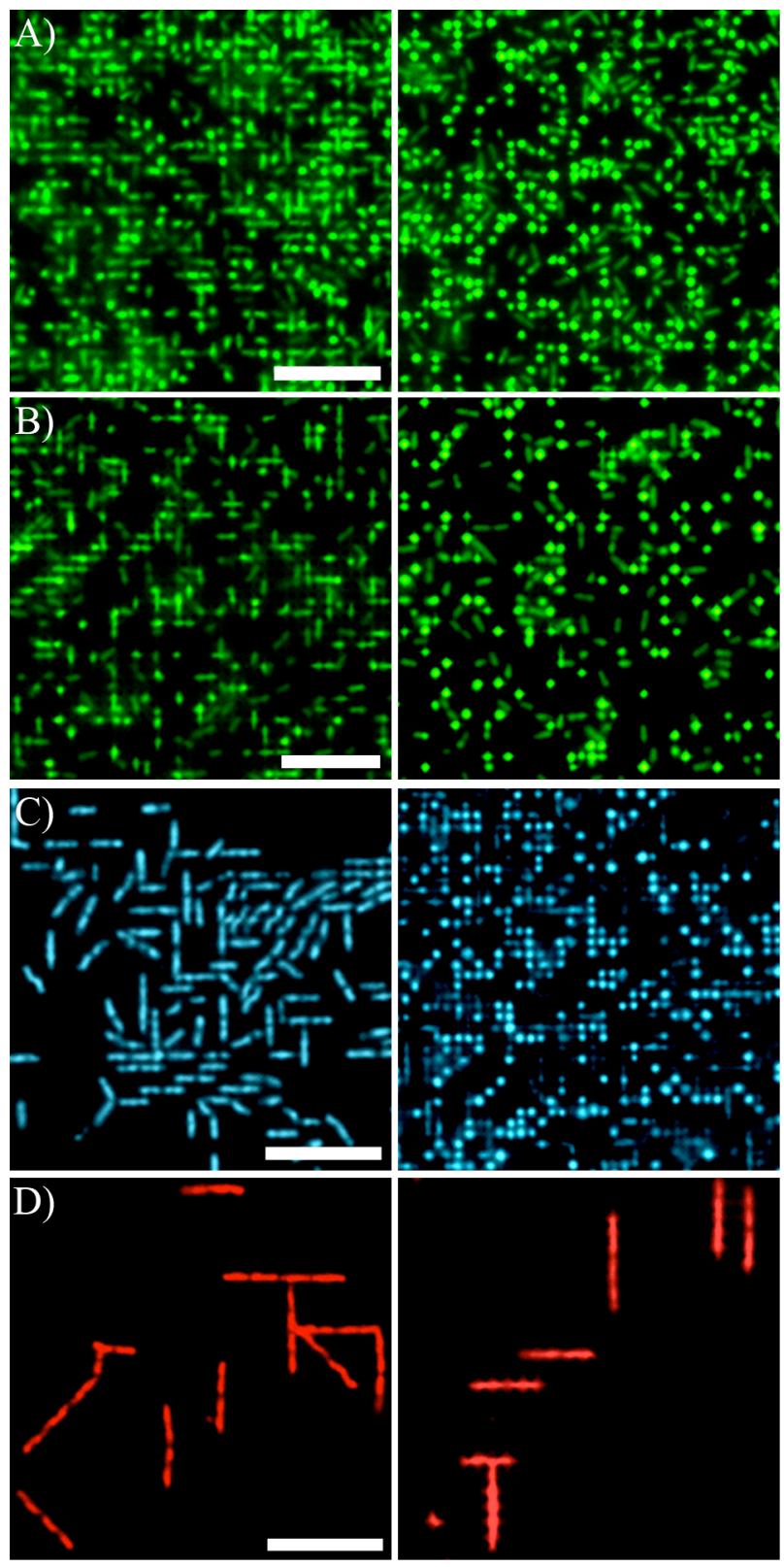

Figure 4. Bacterial assembly on periodic nanopost arrays is a general phenomenon. The fluorescence microscopy images of pili (A) and flagella (B) mutants of $P$. aeruginosa grown on these submerged substrates show that patterning is persistent even in strains lacking the appendages typically used for surface attachment. Spontaneous assembly is also observed in B. subtilis $(\mathbf{C})$ and E. Coli (D) cultured on these surfaces, though at a different lengthscale corresponding to the differences in the dimensions on the cells. The post spacing is wider in the left image and narrower in the image on the right for $\mathbf{A}$ ) through D). All cells were labeled with SYTOX green nucleic acid stain and B. subtilis and E. Coli are 
false-colored blue and red, respectively. Scale bars are all $10 \mu \mathrm{m}$ and apply to the left and right images.

\section{References}

1. Klevens, R. M.; Edwards, J. R.; Richards, C. L.; Horan, T. C.; Gaynes, R. P.; Pollock, D. A.; Cardo, D. M. Public Health Reports 2007, 122 (2), 160-166.

2. Costerton, J. W.; Stewart, P. S.; Greenberg, E. P. Science 1999, 284 (5418), 1318-1322.

3. Vincent, J. L.; Sakr, Y.; Sprung, C. L.; Ranieri, V. M.; Reinhart, K.; Gerlach, H.; Moreno, R.; Carlet, J.; Le Gall, J. R.; Payen, D.; Pati, S. O. A. I. Critical Care Medicine 2006, 34 (2), 344-353.

4. Gristina, A. G. Science 1987, 237 (4822), 1588-1595.

5. Bos, R.; van der Mei, H. C.; Busscher, H. J. Fems Microbiology Reviews 1999, 23 (2), 179-230.

6. Neu, T. R. Microbiological Reviews 1996, 60 (1), 151-166.

7. Schumacher, J. F.; Carman, M. L.; Estes, T. G.; Feinberg, A. W.; Wilson, L. H.; Callow, M. E.; Callow, J. A.; Finlay, J. A.; Brennan, A. B. Biofouling 2007, 23 (1), 55-62.

8. Stevens, M. M.; George, J. H. Science 2005, 310 (5751), 1135-1138.

9. Discher, D. E.; Janmey, P.; Wang, Y. L. Science 2005, 310 (5751), 1139-1143.

10. Huebsch, N.; Arany, P. R.; Mao, A. S.; Shvartsman, D.; Ali, O. A.; Bencherif, S. A.; RiveraFeliciano, J.; Mooney, D. J. Nature Materials 2010, 9 (6), 518-526.

11. Ingber, D. E. International Journal of Developmental Biology 2006, 50 (2-3), 255-266.

12. Engler, A. J.; Sen, S.; Sweeney, H. L.; Discher, D. E. Cell 2006, 126 (4), 677-689.

13. Davey, M. E.; O'toole, G. A. Microbiology and Molecular Biology Reviews 2000, 64 (4), $847-$ 867. 
14. Lichter, J. A.; Thompson, M. T.; Delgadillo, M.; Nishikawa, T.; Rubner, M. F.; Van Vliet, K. J. Biomacromolecules 2008, 9 (6), 1571-1578.

15. Diaz, C.; Schilardi, P. L.; Claro, P. C. D.; Salvarezza, R. C.; de Mele, M. A. F. L. Acs Applied Materials \& Interfaces 2009, 1 (1), 136-143.

16. Ben-Jacob, E.; Cohen, I.; Gutnick, D. L. Annual Review of Microbiology 1998, 52, 779-806.

17. Klausen, M.; Aaes-Jorgensen, A.; Molin, S.; Tolker-Nielsen, T. Molecular Microbiology 2003, $50(1), 61-68$.

18. Klausen, M.; Heydorn, A.; Ragas, P.; Lambertsen, L.; Aaes-Jorgensen, A.; Molin, S.; TolkerNielsen, T. Molecular Microbiology 2003, 48 (6), 1511-1524.

19. Lenz, A. P.; Williamson, K. S.; Pitts, B.; Stewart, P. S.; Franklin, M. J. Applied and Environmental Microbiology 2008, 74 (14), 4463-4471.

20. Vlamakis, H.; Aguilar, C.; Losick, R.; Kolter, R. Genes \& Development 2008, 22 (7), 945-953.

21. Stewart, P. S.; Franklin, M. J. Nature Reviews Microbiology 2008, 6 (3), 199-210.

22. Blango, M. G.; Mulvey, M. A. Current Opinion in Microbiology 2009, 12 (2), 177-181.

23. Shapiro, L.; McAdams, H. H.; Losick, R. Science 2002, 298 (5600), 1942-1946.

24. Weibel, D. B.; DiLuzio, W. R.; Whitesides, G. M. Nature Reviews Microbiology 2007, 5 (3), 209-218.

25. Takeuchi, S.; DiLuzio, W. R.; Weibel, D. B.; Whitesides, G. M. Nano Letters 2005, 5 (9), 18191823.

26. Kastrup, C. J.; Boedicker, J. Q.; Pomerantsev, A. P.; Moayeri, M.; Bian, Y.; Pompano, R. R.; Kline, T. R.; Sylvestre, P.; Shen, F.; Leppla, S. H.; Tang, W. J.; Ismagilov, R. F. Nature Chemical 
Biology 2008, 4 (12), 742-750.

27. Shen, F.; Pompano, R. R.; Kastrup, C. J.; Ismagilov, R. F. Biophysical Journal 2009, 97 (8), 2137-2145.

28. Pokroy, B.; Epstein, A. K.; Persson-Gulda, M. C. M.; Aizenberg, J. Advanced Materials 2009, 21 (4), 463-469.

SYNOPSIS TOC

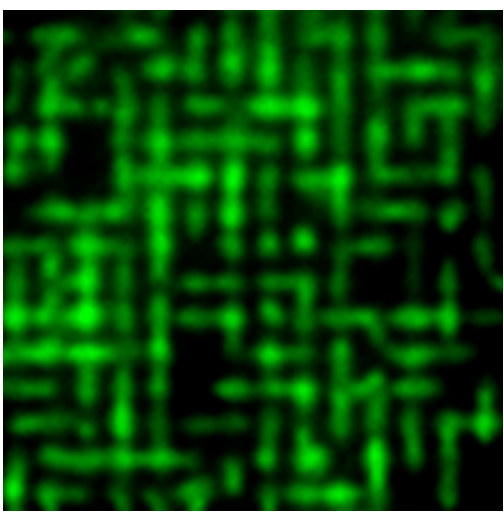

